\title{
Diferenciación de especies Diatraea spp. que afectan los cultivo de caña en la región del Gualiva
}

\section{Species Differenciation of Diatraea spp, which affect the sugarcane crops in Gualivá, Colombia}

\author{
Sandra Parada \\ Tesista. Ingeniería Agroecológia. Corporación Universitaria Minuto de Dios. \\ agrosandraparada@yahoo.es \\ Eberth Ebratt \\ Msc. Entomólogo Laboratorio de Diagnóstico \\ Fitosanitario y Molecular ICA Tibaitata. \\ evebatt@yahoo.com \\ Miguel A. Benavides \\ Magíster en entomología, ingeniero agrónomo de la Universidad de Nariño, \\ docente de cátedra de la Corporación Universitaria Minuto de Dios del programa \\ de Ingeniero de Sistemas desde el año 2004. \\ mbenavides@uniminuto.edu
}

\section{Resumen}

Se realizó un diagnóstico de las especies de Diatraea spp, encontradas durante la recolección directa de estados inmaduros en cañas listas para beneficio y cañas de 7 a 9 meses después del corte. La recolección de los espécimenes, se hizo directamente sobre los tallos de cañas en cultivos de la variedad POJ en los municipios de Villeta y Nimaima Cundinamarca, por un tiempo de ocho meses empezando en el mes de Octubre de 2006 y finalizando en el mes de Junio de 2007, con un total de ochenta larvas recolectadas.

Se reconocieron tres especies de barrenador Diatraea saccharalis, D. indigenella y una especie que se encuentra en proceso de reconocimiento; en la recolección se obtuvieron promedios de porcentaje de $75,71 \%$, 22,81 y 1,44\%, respectivamente. El diagnóstico consistió en observar las diferencias morfológicas mas relevantes de cada una de las tres especies.

Palabras claves. Barrenador de la caña, Diatraea saccharalis, D. indigenella, Diatraea spp, Diagnosis, características morfológicas, setas, pináculas, mandíbulas.

\section{Abstract}

An analysis was carried out from the species of Diatraea spp, that were found during the direct recollection from unripened sugarcanes that were ready for benefit and canes from 7-9 months after the harvest. The gathering from the specimens was made directly over the sugarcanes stalks (POJ variety cultivations) in the municipalities of Villeta and Nimaima, Cundinamarca for a period of eight months, beginning in October 2006 and ending in June 2007. The total number of larvae gathered was eighty.

Three borer species were identified: Diatraea saccharalis; D. indigenella and one specie that is to be identified. The average percentages in the gathering were $75.71 \%, 22.81 \%$ and $1.44 \%$ respectively. The analysis consisted of observing the most important morphological differences among these three species.

Keywords. Cane borer, Diatraea saccharalis, D. indigenella, Diatraea spp, diagnosis, morphological characteristic, seta, pinacula, jaws. 


\section{Introducción}

El barrenador de la caña Diatraea spp, es la especie mas limitante dentro del cultivo ya que sus perjuicios ocasionan pérdidas considerables en la producción como reducción en la cantidad de jugos, dependiendo de la severidad y los daños que se originen en campo como lo son: perforaciones en los entrenudos del tallo de la caña, formación de galerías por el consumo de la larva, entrada de patógenos y de otros insectos que causan mayor detrimento a la caña. (Benavides, Mora. 2004)

Los estudios que se han realizado con respecto a la diferenciación de especies de Diatraea se enfocan a su identificación por medo del reconocimiento de espécimenes en estado adulto, por medio de la extracción del aparato genital de los individuos, o se han tenido en cuenta los restados en estados inmaduros como pupa y en un estudio realizado por Pastrana (1991) en el que se da a conocer el ciclo biológico de D. saccharalis comparado con D. indigenella el cual especifica cada una de las diferencias en los diferentes estados del insecto.

Según Solís (2005), se encontraron diferencias significativas entre D. saccharalis y D. lineolata. En las setas paraproctales, ya que se diferencian en el tamaño, para D. saccharalis se presentan de igual tamaño, y para D. lineolata se diferencian, ya que la interna es $1 / 3$ la seta externa ${ }^{1}$.

Este trabajo fue realizado en la región de Gualiva, durante diez (10) meses de recolección de larvas, directamente de los tallos de cañas en un periodo de desarrollo de 8 a 9 meses, iniciando en el mes de octubre de 2006 hasta el mes de junio de 2007.

La región del Gualiva es de gran importancia dentro de la producción de caña panelera en Cundinamarca siendo el mayor productor de panela en

${ }^{1}$ La característica de D. lineolata se confunde fácilmente con D. spp cerca de grandiosella. el departamento con producciones de 4,4 tn/ ha de panela (Agrocadenas 2005).

A causa de la importancia de la plaga y la incidencia de nuevas especies del género se propuso realizar un reconocimiento e identificación de las especies de Diatraea que actualmente se encuentran atacando los cultivos de caña panelera en la región, identificando las características más representativas en cada una de las especies.

Dentro de los resultados se encontraron diferencias significativas, entre las especies en el estado de larva. Se obtuvo un espécimen que al parecer no se encuentra reportado en el país atacando gramineas.

Este individuo está en proceso de identificación por parte de un especialista en el genero Diatraea, igualmente se darán a conocer las características mas representativas de las especies encontradas.

\section{Área de estudio}

Dentro de los municipios productores encontramos a Villeta con un total de área sembrada en caña panelera de 5.600 hás. y Nimaima con un total de 2.300 hás.; estos dos municipios fueron el objeto de estudio (Anuario, 2006).

Villeta por ser el municipio central en comercialización y de mayor área sembrada en Cundinamarca, se encuentra entre los 850 y 1200 m.sn. $\mathrm{m}$, con temperaturas de 18 a $25^{\circ} \mathrm{C}$ y precipitación promedio de $1600 \mathrm{~mm} /$ año (sitio web del municipio).

El municipio de Nimaima en evaluaciones previas presentó un alto índice de severidad, por ende fue importante la muestras recolectadas. Este municipio se encuentra a una altura promedio de 1185 m.s.n.m con una temperatura promedio de $24^{\circ} \mathrm{C}$ (sitio web del municipio), y su 
rendimiento está por el margen de 3 Tn / ha. (Anuario, 2006)

Las variedades que se encuentra en producción son la variedad POJ 2878 de mayor resistencia al barrenador y la variedad ZC susceptible al barrenador y al carbón de la caña, pero mucho más productiva, lo que significa que su periodo vegetativo es más corto aproximadamente de 10 a 12 meses, mientras que la variedad POJ 2878 puede durar hasta 18 meses en desarrollo, (información de productores).

Una de las actividades mas representativas dentro del cultivo es el sistema de corte por entresaque que consiste en cortar sólo las cañas maduras, dejando así plantas para un nuevo corte $\mathrm{y}$, de esta manera, no hacer tan prolongado el tiempo entre cosechas con la finalidad de obtener cañas periódicamente para la comercialización.

Esta situación favorece el establecimiento de la plaga.

\section{Objetivo de la investigación}

El objetivo principal de esta investigación es dar a conocer las características más representativas de las especies de Diatraea spp encontradas durante la recolección en la región del Gualiva.

\section{Metodología}

Se escogieron nueve (9) fincas dentro de los dos municipios ubicadas en seis (6) veredas (La Mazata, Bagazal, Alto de Torres, Nimaima, Mabe y La Calera). Estas veredas se localizaron geográficamente por medio de un GPS, los datos de ubicación se muestran en la Tabla 1.

Para la recolección se buscaron lotes donde recién se hubiera realizado la cosecha, para una mayor facilidad en la ubicación de los especimenes.
Para encontrar al insecto en estado de larva, se realizó una observación directa sobre los tallos de la caña, ubicando orificios recientes de entrada de la larva, los cuales se reconocieron por los desechos o defecación de las larvas por la barrenación.

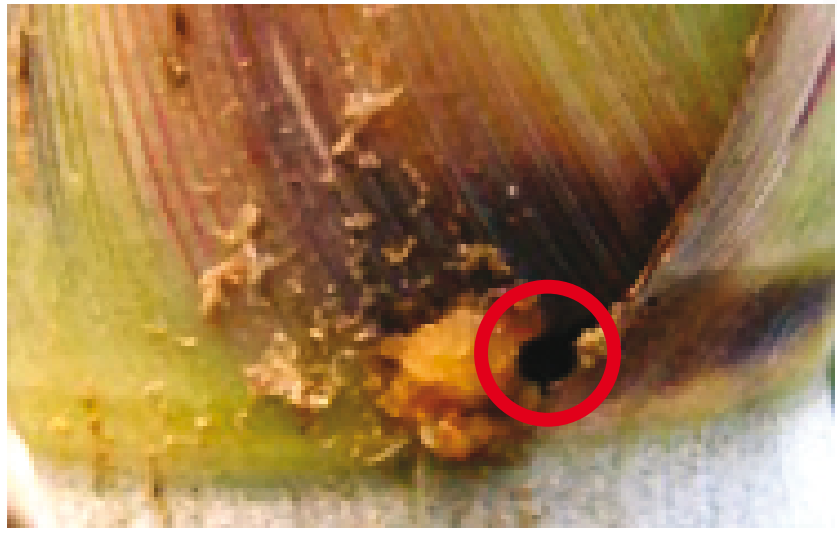

Figura 1. Perforación de entrenudos

Una vez ubicado el (los) orificio(s) se extrajo la caña de la cepa y se realizo un corte transversal del tallo, teniendo en cuenta de no dañar el espécimen; para encontrar el estado inmaduro se cortó un entrenudo arriba y debajo de la ubicación de la larva y se selló la caña para ser transportada a laboratorio y conservarlas vivas.

\begin{tabular}{|c|c|c|c|c|}
\hline HIPla: a & Éトд & VAK トIJAI! & msnm & 'J-HトIIA \\
\hline Dejilkn Cài & $27 \mathrm{fo}$ & $\mathrm{POJ}, 2 \mathrm{C}$ & $\$ 00$ & $\begin{array}{l}\text { doto de } \\
\text { lories }\end{array}$ \\
\hline 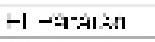 & : 4 I I I & ¿C: $ト$ III & Mr & Нનુja: \\
\hline a Parcre & S ha & rod, zC & $\cos$ & Mets \\
\hline - I -ariche & 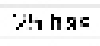 & 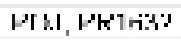 & $11 \%$ & I $7 \mathrm{hda79s}$ \\
\hline コT-apiche & $1: \mathrm{se}$ & ro.l & $10: 54$ & Ls Narats \\
\hline -t. n7 וn & $\begin{array}{l}\text { ak } \\
\text { rasi }\end{array}$ & Fin. FR $7:$ & in:"it & 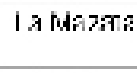 \\
\hline ヨ ニ & 70 hac & $\begin{array}{l}\text { POJ, } \\
\text { Manuolta, } \\
\text { 2r. Pulmir: }\end{array}$ & 1103 & Lu Celara \\
\hline$\exists=$ = du & $\varepsilon$ lad & POJ, $2 C$ & 803 & Idr Idil|le \\
\hline Sacr3 Pack & $a t=$ & $\mathrm{POJ}, \mathrm{ZC}$ & 1103 & $\begin{array}{l}\text { Cusz.aca } \\
\text { Hวาda }\end{array}$ \\
\hline
\end{tabular}

Tabla 1. Fincas de recolección

\section{Metodología}

Ya en el laboratorio se clasifican las muestras por fincas, se buscan las larvas dentro de la caña y se someten a desnaturalización en agua (esto es introducir las larvas en ebullición a $100^{\circ} \mathrm{C}$, apagar y 
dejar alli un promedio de 10 minutos, siendo mas fácil su conservación) luego estas se llevan a conservación en alcohol al 70\%, en tubos de ensayo.

La identificación se realizó por diferentes procesos para facilitar la toma de datos. Entre las cuales están: a) envío de muestras a Palmira de dos especies de Diatraea, D. saccharalis y D. indigenella. b) Determinación de sus diferencias morfológicas, como mapa setal, color, pináculas y aparato bucal (mandibulas); para este último se extrajeron las mandíbulas con la ayuda de pinzas y una punta o canutillo, se extrajo la mandíbula izquierda de cada una de las muestras y posteriormente se aclararon y se limpiaron en una solución de hidróxido de potasio $(\mathrm{KOH})$, por un tiempo de dos horas, se introdujeron en un tubo de ensayo a baño María. c) Una vez identificadas las diferencias entre estas dos especies se procedió a la comparación de las larvas recolectadas. d) Uso de claves taxonómicas para corroborar las diferencias entre especies.

El reconocimiento de las larvas se realizó bajo un estereoscopio de alta definición, allí se observaron diferencias suficientes para la identificación de Diatraea en estado larval.

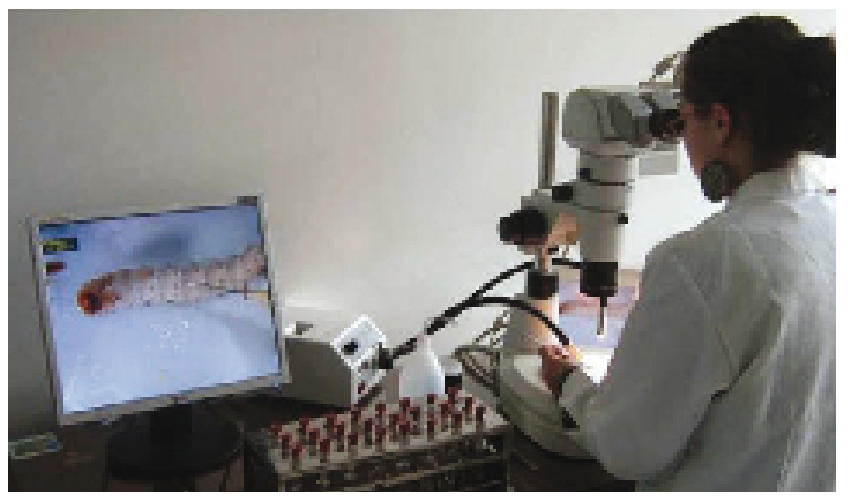

Figura 2. Identificación con observaciones al estereoscopio.

\section{Resultados}

Las especies encontradas en la zona del Gualiva pertenecen a cultivos de caña panelera de 6 a 7 meses de crecimiento después del corte, o de cañas listas para beneficio sometidas a muestreo durante 10 meses, empezando en el mes de mayo de 2006 y finalizando el mes de junio de 2007, con un total de 105 larvas.

Los porcentajes encontrados para cada una de las se muestran en la gráfica 1 .

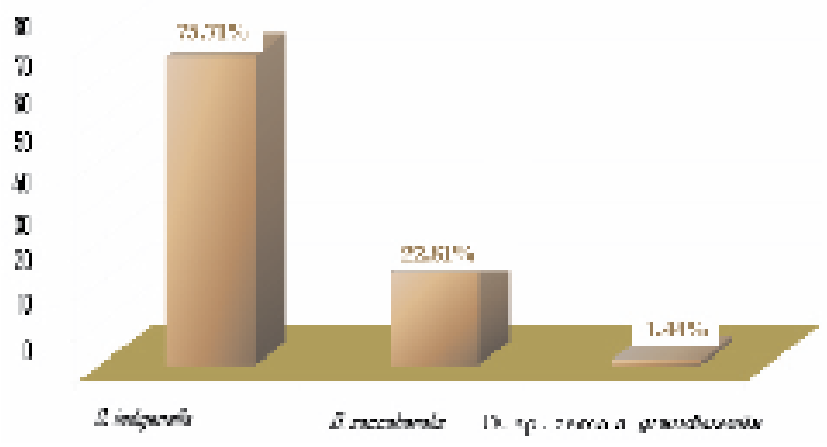

Gráfica 1. Porcentajes de especies encontradas

En la gráfica 1 se puede observar que la D. indigenella es la especie que predomina dentro del ataque de la caña panelera con el mayor porcentaje de espécimenes encontrados, seguida de D. saccharalis, esto da un giro en lo que se venía planteando por los técnicos de la región ya que se pensaba que sólo existía la presencia de esta ultima especie El último porcentaje se da para una especie que aun está en proceso de reconocimiento por parte de un especialista, según la identificación realizada en el laboratorio sus características son similares a las de la especie D. Grandiosella, no identificada en el país.

A continuación, se describirán las características morfológicas representativas de las especies encontradas.

\section{- Diatraea saccharalis}

En general esta larva es de color blanco crema con panículas de color amarillo, y presencia de pequeñas tonofibrillas de igual color pero de menor tamaño, que hacen la identificación más fácil. 


\section{Cabeza}

Presenta una cabeza normal de lepidóptero, de color café brillante a rojo. Presenta chaetotaxia normal de lepidóptero. Alrededor de la sutura epicraneal y de la sutura adfrontral una aclaración o linea mas clara formando una $\mathrm{Y}$ en vista dorsal, además estas suturas presentan un color café oscuro notorio en vista dorsal o pleural.

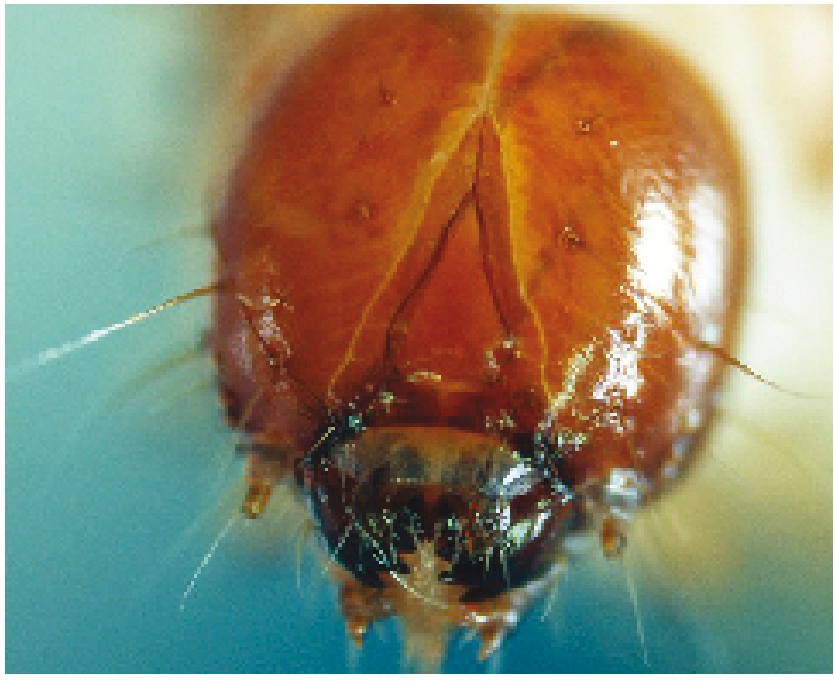

Figura 3. Cabeza de D. saccharalis

\section{Escudo protoráxico}

Presenta un color café rojizo claro con manchas más oscuras no secuenciales, posteriores a las setas anteriores y las setas posteriores que conforman el escudo, el número de setas es de doce con diferentes tamaños, siendo más largas las anteriores o más cercanas a la cabeza.

\section{Mandíbulas}

Exteriormente son de color café oscuro más oscuro que la cabeza. Presenta 4 dientes punteagudos y con un diente más pequeño en el exterior interno de la mandibula; son bastante fuertes y quitinizadas. Su longitud es de 0.58 y su anchura es 0.47 micras un medido a la parte mayor.

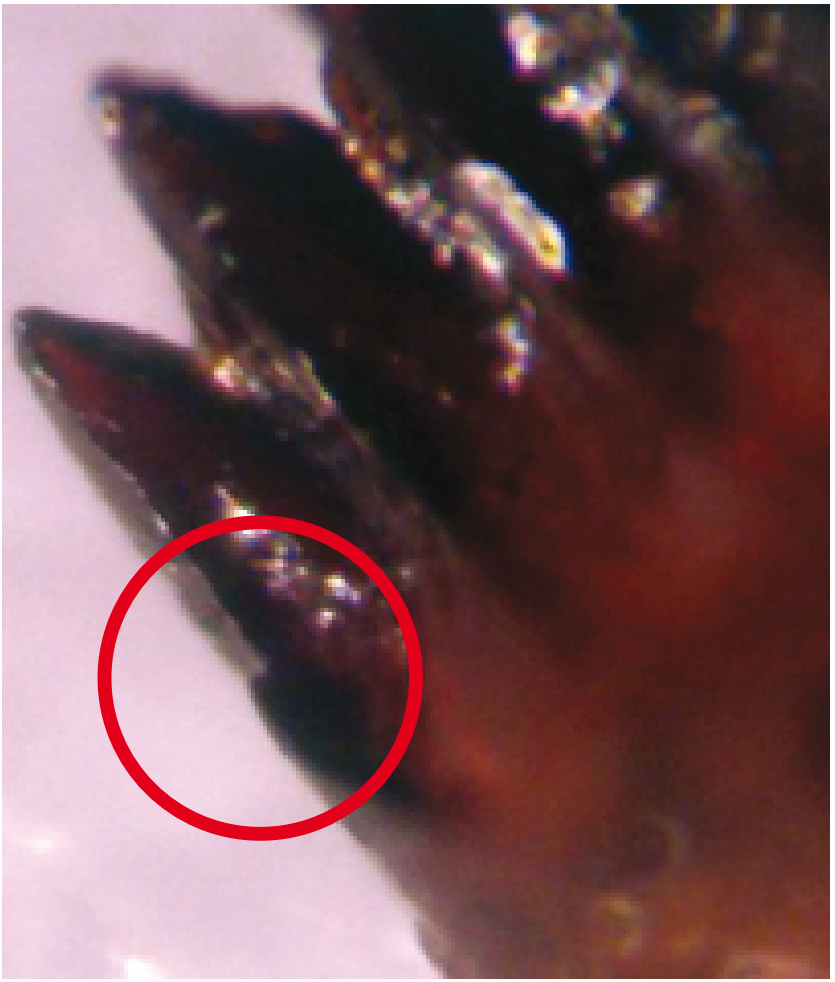

Figura.4. Mandibula de D. saccharalis. Diente característico.

\section{Sección dorsal}

Presenta pináculas de color amarillo de forma circular y regular con una seta por cada una, en cada segmento presenta tonofibrillas que igualmente están rodeadas de un círculo amarillo pero de menor tamaño sobre las panículas anteriores del dorso. Por cada segmento presenta cuatro panículas bisetosas y dos tonofibrillas.

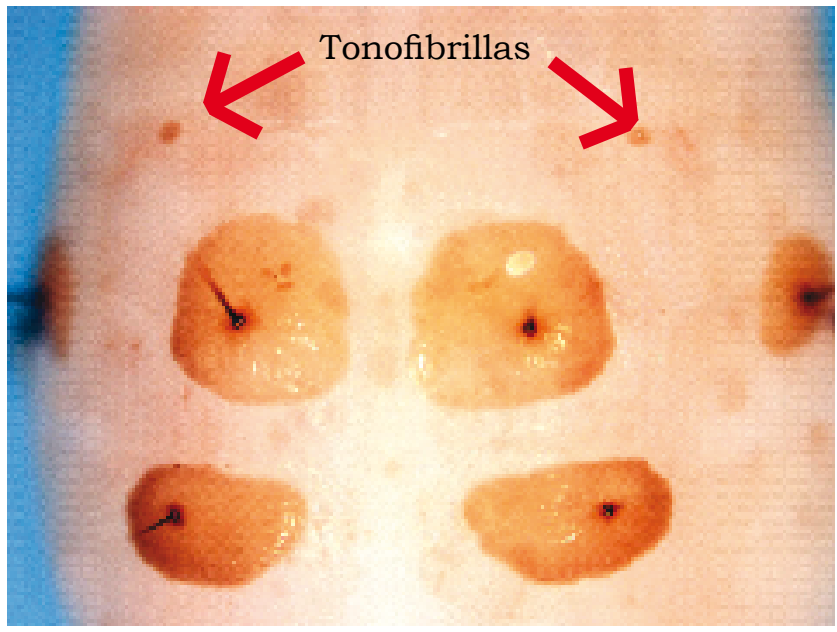

Figura 5. Pináculas y Tonofibrillas dorsales en D. saccharalis 


\section{Sección lateral}

Esta sección presenta espiráculos en los segmentos $\mathrm{T} 1, \mathrm{~A} 1-\mathrm{T} 8$, son de forma ovalada y presentan un recubrimiento esclerotizado o un borde más delgado, anterior al espiráculo se observa una tonofibrilla que se encuentra también dentro de una pinácula o un círculo de color amarillo que se observa fácilmente en el estereoscopio. Esta característica es importante para la identificación de especies, ya que es más notoria en D. saccharalis que para otras especies del género. Igualmente presenta pináculos redondeados de forma regular; en la parte lateral se pueden observar tres pináculas una latero-dorsal o sobre el espiráculo y otra lateral o debajo del espiráculo cada una bisetosa y una latero-ventral tricetosa.

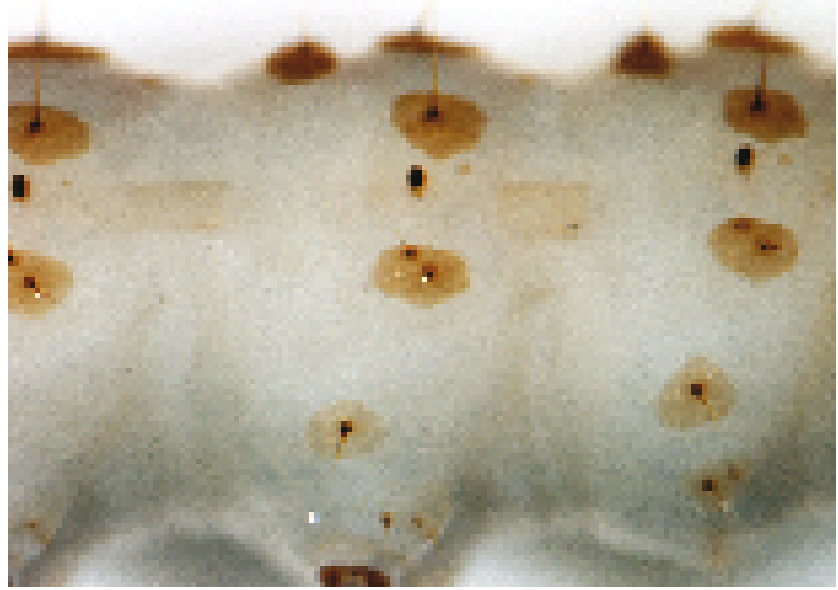

Figura.6. Segmento A6 vista lateral

\section{Sección ventral}

Está conformada por las patas verdaderas y las seudopatas centrales y anales, en estas se encuentra una característica representativa para la especie presenta un tipo de setas denominadas paraproctales, que para D. saccharalis son de igual tamaño a diferencia de otras especies en su género.

En esta sección podemos encontrar tonofibrillas formando cuatro triángulos ubicados entre los segmentos T2 y T3 que igualmente están ro- deados de una pinácula de menor tamaño que las laterales y dorsales, característica a tener en cuenta en la identificación.

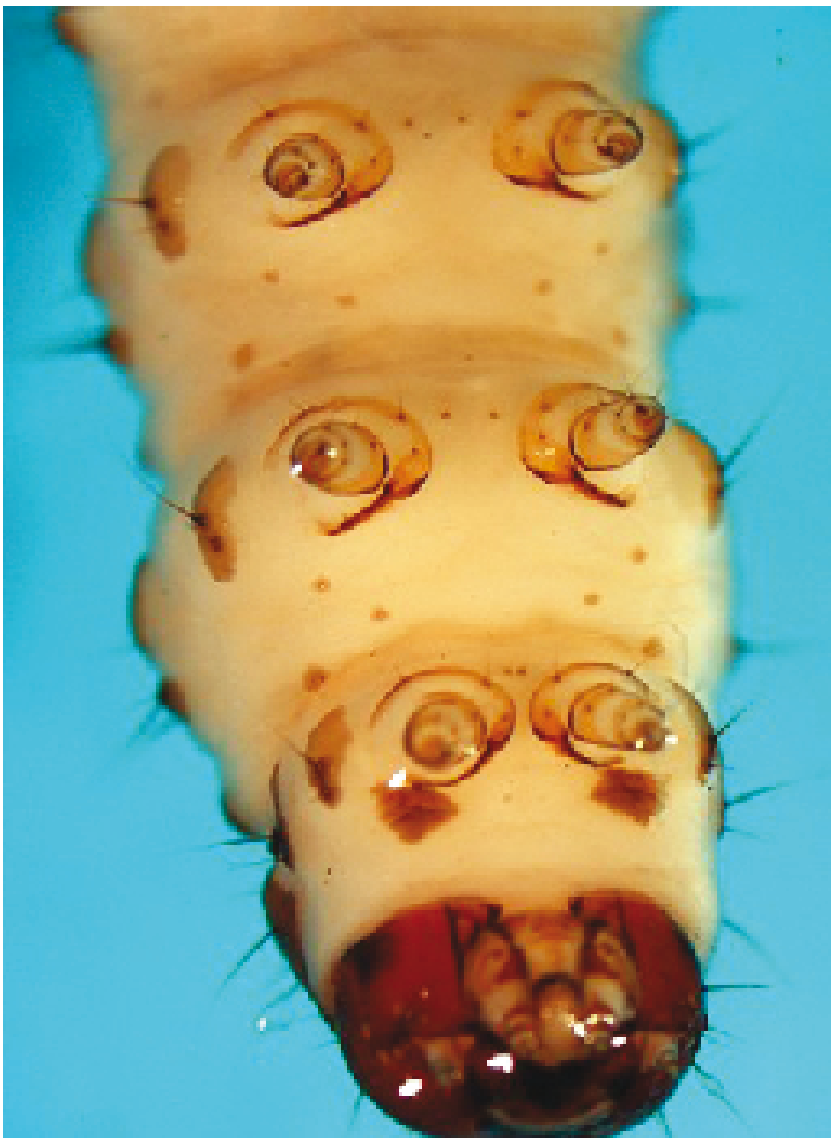

Figura 7. Segmentos T2 y T3

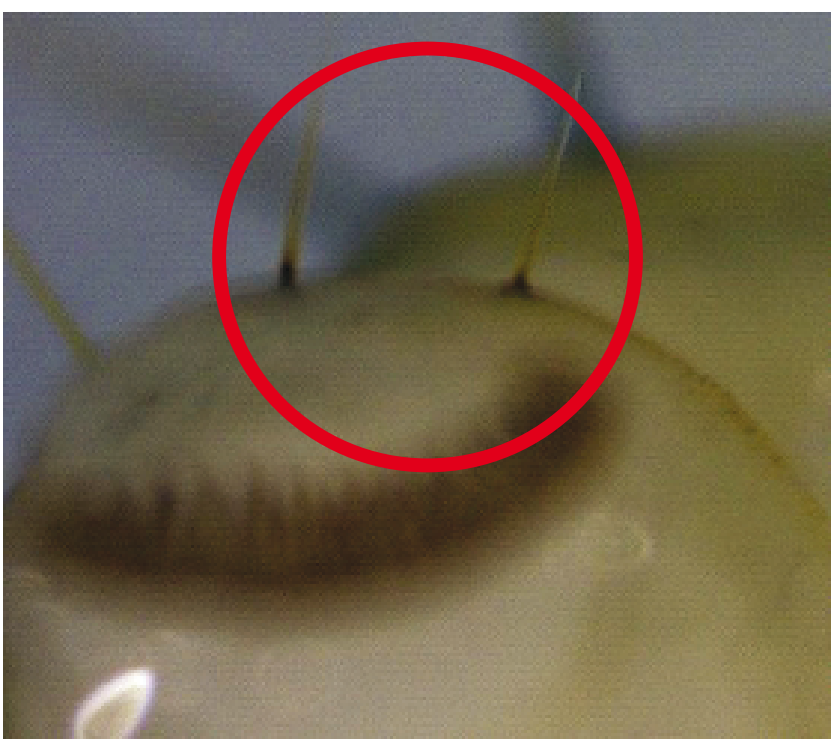

Figura 8. Setas paraproctales 
Las seudopatas son de forma circular y poseen crochets o espinillas ubicadas de manera triordinal o biordinal, estas se ubican en los segmentos de A3 a A6 y las seudopatas anales son de forma de penielipse, igualmente triordinales o biordinales.
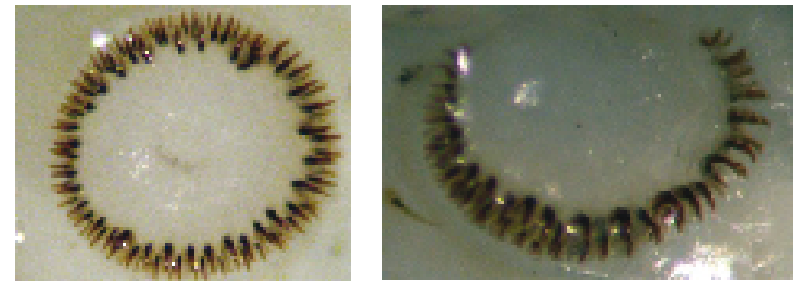

Figura 9. Seudopatas A3-A6. Seudopatas anales.

\section{- Diatraea indigenella}

En términos generales es una larva blanco amarillenta, con manchas de color morado, sus panículas se confunden con las manchas moradas, pues son mas claras. Su cabeza es grande y de color café claro.

\section{Cabeza}

Igualmente presenta una chaetotaxia normal de lepidóptero, su color es más claro y de mayor tamaño que el de D. saccharalis. Presenta un color más claro alrededor de la sutura adfrontal, formando una $\mathrm{V}$ de vista dorsal.

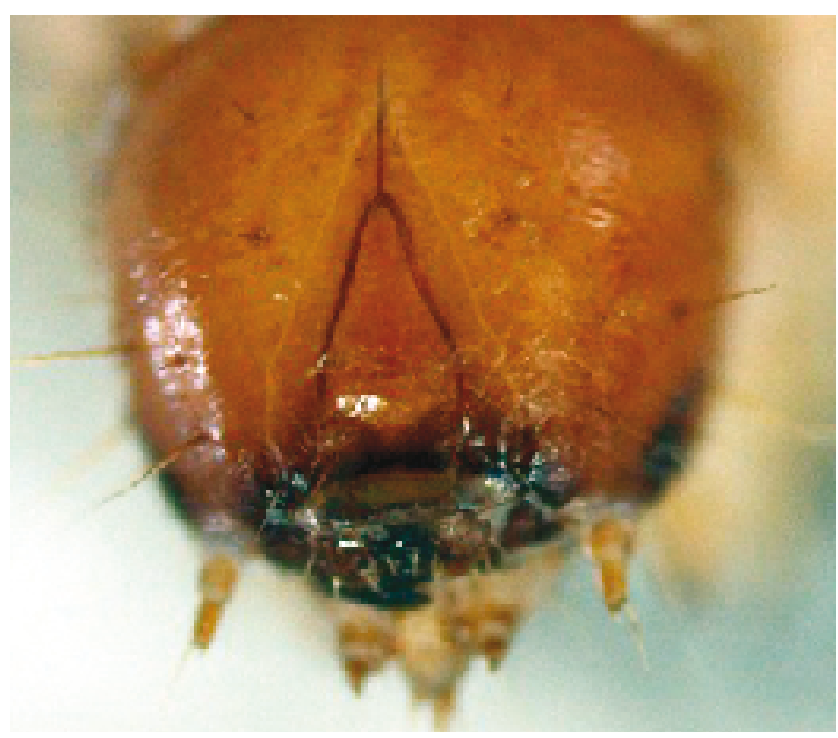

Figura 10. Cabeza de D. indigenella

\section{Escudo protoráxico}

Se encuentra provisto de doce setas de diferente tamaño (seis por cada lado), es de color café claro, con áreas más claras al centro del escudo.

Mandíbulas

Estas son de color café oscuro, posee dientes de punta roma, de gran tamaño; a su vez, tienen un diente adicional pero es más parecido a una protuberancia irregular.

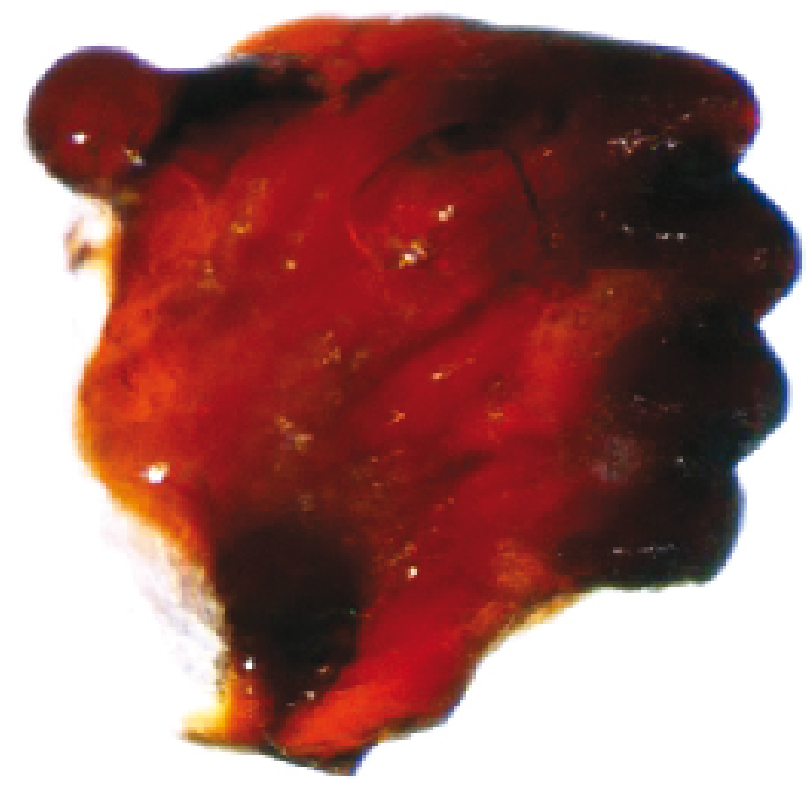

Figura 11 . Mandibula de D. indigenella

\section{Sección dorsal}

Presenta panículas bisetosas (T1 y T2) y unisetosas (T3- A10) de forma irregular de color café claro (4 por segmento) de gran tamaño, presenta tonofibrillas que no se encuentran rodeadas de pequeñas panículas, lo cual las hace dificiles de observar al estereoscopio, esto la diferencia notoriamente de la especie $D$. saccharalis.

Esta especie presenta un color crema con manchas acentuadas de color morado que pueden ir desde T2 hasta A10. 


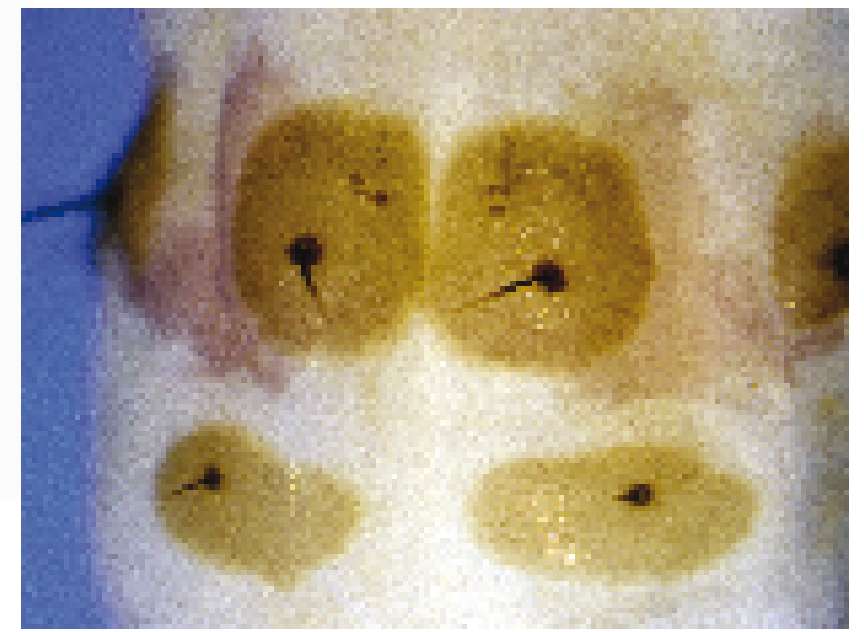

Figura 12. Pináculas dorsales en D. indigenella.

\section{Sección lateral}

Esta sección presenta espiráculos de forma ovalada donde se pueden ver dos secciones una interna más oscura con rugosidades, y una externa esclerotizada y más delgada, estos son de color café oscuro presentes en los segmentos T1, A1-A8. En su parte anterior o en dirección a la cabeza se observan tonofibrillas que son muy dificil de observar al estereoscopio, para poderlas visualizar se debe manejar diferentes adaptaciones del lente.

Esto hace más fácil el reconocimiento frente a otras especies. Se presentan tres panículas una sobre el espiráculo Latero- dorsal unisetosa, otra lateral debajo del espiráculo bisetosa y, por último, una latero- ventral bisetosa desde A1 a

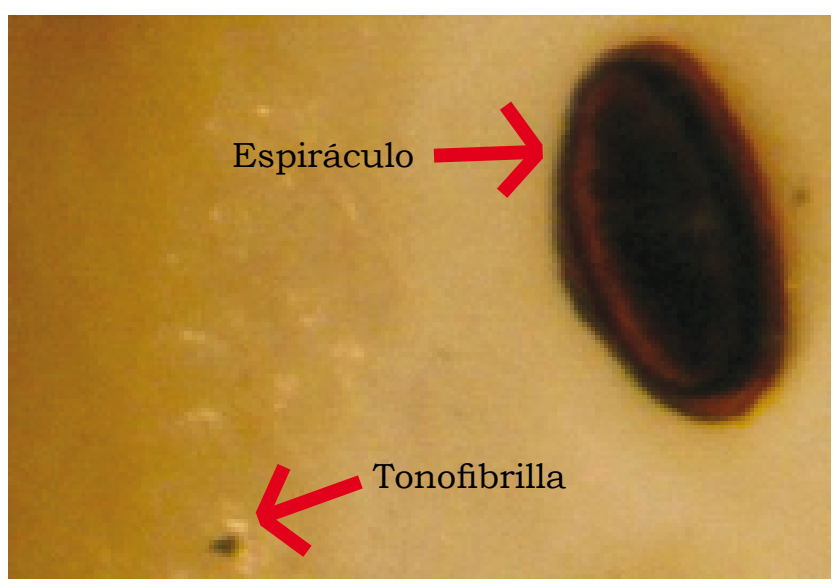

Figura 13. Tonofibrilla lateral y espiráculo
A9. Para T1 el espiráculo se encuentra debajo del escudo protoráxico y dos pináculas bisetosas debajo del espiráculo. Para T2 y T3 no hay presencia de espiráculos pero se observan cuatro pináculos, tres de los cuales son bisetosos y un unisetoso.

\section{Sección ventral}

Está conformada por las patas verdaderas y las seudopatas centrales y anales, en estas últimas se encuentran las setas paraproctales que presentan un tamaño diferente siendo las externas de mayor tamaño que las internas.

A diferencia de D. saccharalis, D. indigenella no presenta tonofibrillas en los segmentos T2 y T3.

Las seudopatas son triordinales de forma circular en el caso de A3 -A6 y las patas anales igualmente son triordiales pero en forma de penielipse.

\section{- Diatraea sp. cerca a grandiosella}

Esta especie no se encuentra reportada en el país, por ello la importancia de su identificación; del $100 \%$ de las larvas recolectadas sólo un 1\% es D. grandiosella, la característica por la cual se dice que esta especie es de las seta paraproctales pues según Coto (1997), presenta la seta interna en forma de tonofibrilla y bastante pubescencia en la zona anal.

\section{Cabeza}

Es de color café rojizo a negro, presenta una chaetotaxia y estructura normal de lepidóptero.

\section{Mandíbulas. Sin definir. Sección dorsal}

Es una larva de color blanco a amarillo crema, presenta panículas de color café oscuro a negro,

${ }^{2}$ Comparada con D. lineolata esta no posee papila q sostenga la tonofibrilla que forma la seta paraproctal. 


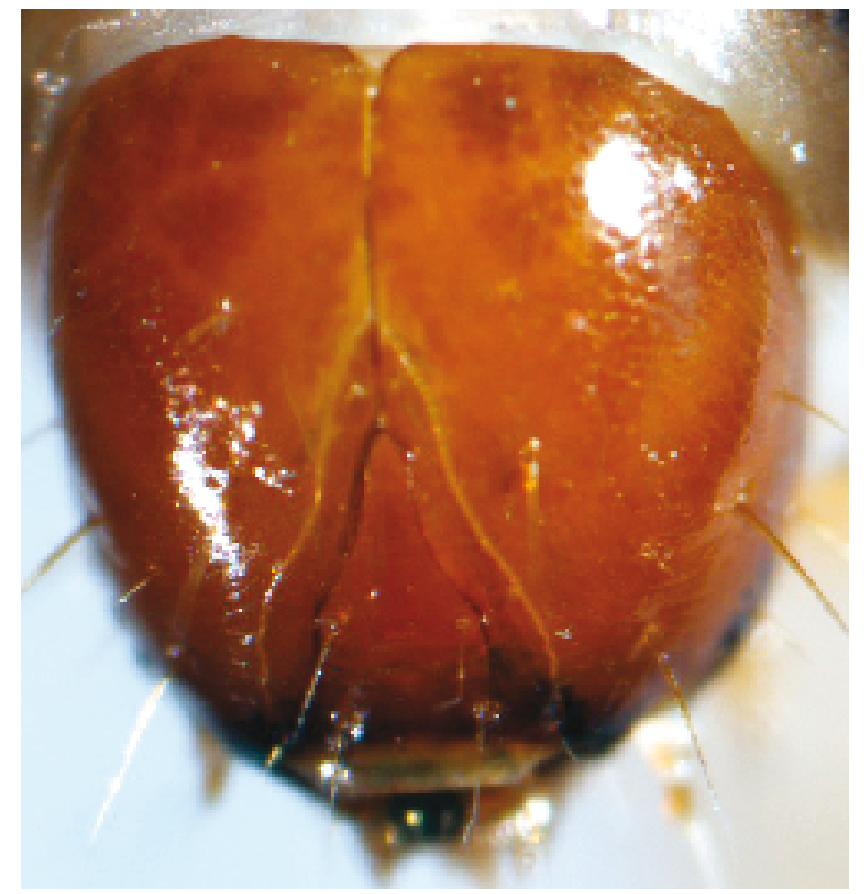

Figura 14. Cabeza de D.sp, cerca a grandiosella

cuatro por cada segmento; estas son de forma una poco irregular, mostrando mayor tamaño las anteriores, no posee tonofibrillas sobre estas panículas. Cada pinácula posee una sola seta. Una diferencia notoria es la presencia de manchas mas claras en cada pinácula alrededor de la seta son pequeños huecos o hundimientos que se observan fácilmente; se advierten entre dos o tres máximo cuatro por pinácula.

\section{Sección lateral}

Tienen una conformación de panículas igual a las especies anteriores; equivalentemente, posee tonofibrillas en la parte anterior del espiráculo pero de menor tamaño, siendo dificiles de observar.

\section{Sección ventral}

Esta presenta la más importante de las características, ya que igual a D. saccharalis muestra el triángulo de tonofibrillas en los segmentos T2 y T3, pero lo más significativo y que hace la diferencia principal es la presencia de una seta a manera de tonofibrilla en las setas paraproctales alojadas en las seudopatas anales; además, de presentar
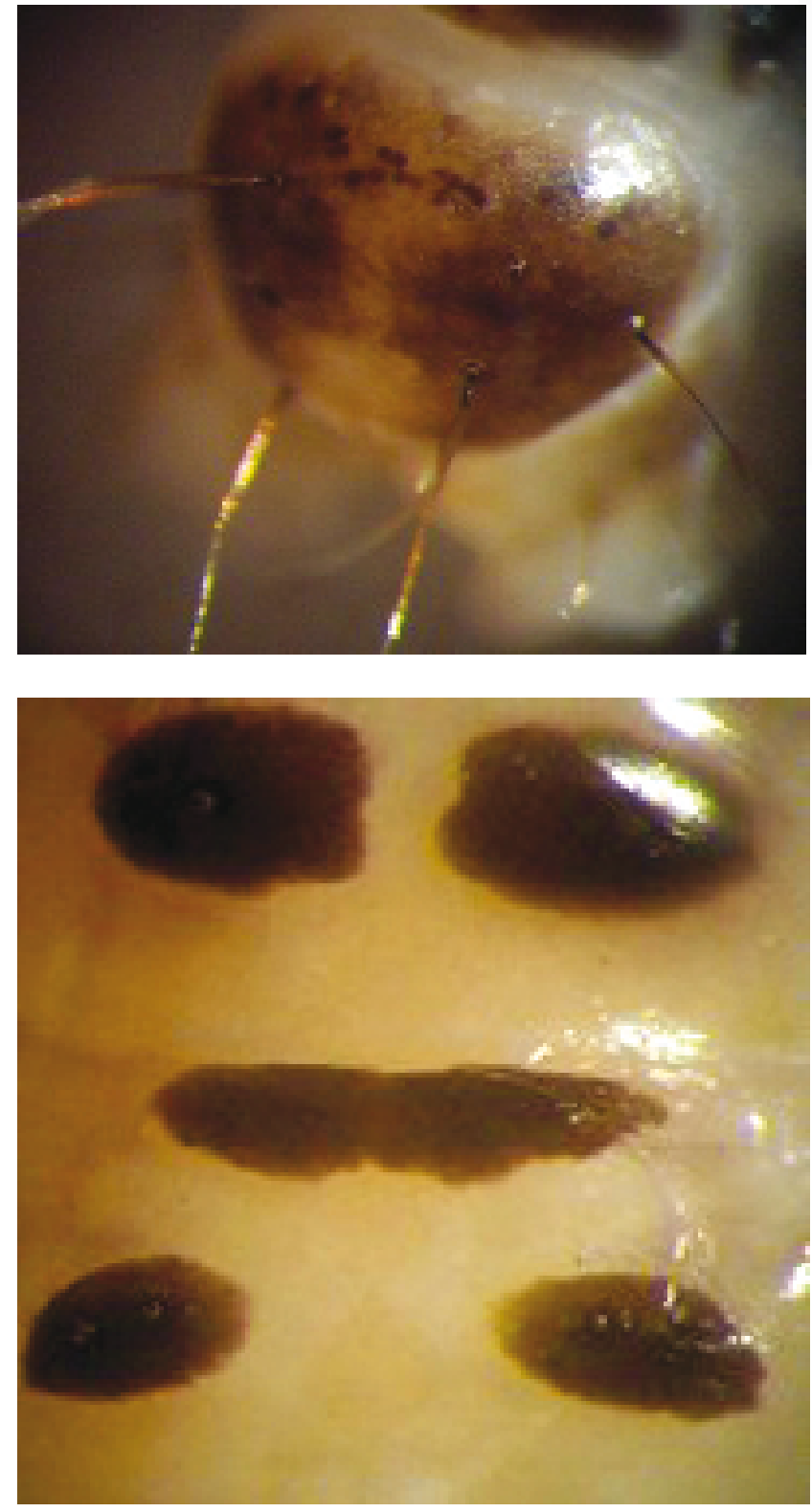

Figura 15-16. Panículas- Escudo anal de D. grandiosella

pubescencia abundante en dicha región.

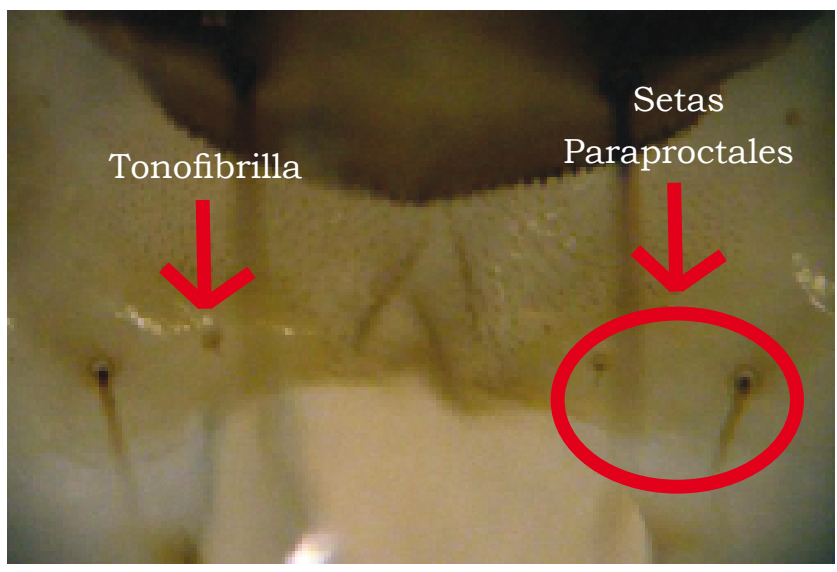

Figura 17. Setas paraproctales de D. grandiosella 


\section{Referencias bibliográficas}

- Coto, D. 1997. Lepidoptera en cultivos anuales y perennes. Manual de reconocimiento. CATIE. Turrialba, Costa Rica.

- Peterson, A. 1951. Larvae of insects. Lepidotera and Hymenoptera. Part I. Columbus. Ohio.

- Solís, A. 1986. Key to selected pyraloidea (lepidoptera) larvae intercepted at u.s. ports of entry: revision of pyraloidea in "keys to some frequently intercepted lepidopterous larvae" by Weisman. National Museum of Natural History. Washington, D.C..

$1^{8}$ - Min Agricultura. 2006. Anuario agropecuario.

- $\quad$ Productores de la zona, Dueños de predios evaluados. Encuestas.

- $\quad$ http://villeta-cundinamarca.gov.co/nuestromunicipio. $\mathrm{shtml}$ ?apc $=\mathrm{m}-\mathrm{f} 1--\& \mathrm{~m}=\mathrm{f}$

- $\quad$ http://es.wikipedia.org/wiki/Nimaima.

Fotografias: SANDRA MILENA PARADA PIRE.

ICA. 2007 